\title{
The Value of Establishing the Quantitative Accuracy of PET/CT Imaging
}

\author{
Paul E. Kinahan ${ }^{1}$, David A. Mankoff ${ }^{2}$, and Hannah M. Linden ${ }^{3}$ \\ ${ }^{1}$ Department of Radiology, University of Washington, Seattle, Washington; ${ }^{2}$ Department of Radiology, University of Pennsylvania, \\ Philadelphia, Pennsylvania; and ${ }^{3}$ Department of Medicine, University of Washington, Seattle, Washington
}

A in 2014, and 1 in 4 deaths in the United States will have been from cancer (1). The reasons for the increasing burden of cancer to society and the meager progress are complex, but one reason is that many cancer standard therapies are ineffective. This is compounded by the increasing difficulty in conducting clinical trials to evaluate new therapies (2).

At the same time, there has been an explosive growth of knowledge of the interactions between many of the genetic drivers of cancer, including oncogenes and tumor suppressors, and insights into the genetic heterogeneity of human tumors that are enabled by exponential improvements in DNA sequencing methods (3). The use of targeted small-molecule anticancer treatments and immunotherapies will likely require diagnostic tests to optimize efficacy (4). For these reasons, molecular imaging characterization of tumors and their habitats is likely to become standard clinical practice in targeted therapies, but for this we need improved tools to help guide drug development, identify critical targets, and determine whether the target or pathway is hit. Such tools will catalyze efforts in precision medicine and help clinicians evaluate choices sooner for individual patients.

\section{See page 1137}

Quantitative imaging with PET combined with CT is a valuable tool for assessment of a tumor's response to therapy and for clinical trials of novel cancer therapies because it can measure functional and molecular changes at multiple tumor sites, with faster and more specific indicators of response than anatomic size changes (5). Success with this approach has been demonstrated using ${ }^{18} \mathrm{~F}-\mathrm{FDG}$ for evaluation of therapy-induced changes in glucose metabolism in lung cancer $(6)$ and other tumors. Results from the NeoALTTO (Neoadjuvant Lapatinib or Trastuzumab Treatment Optimization) trial in breast cancer showed an early predictive value for human epidermal growth factor receptor-directed targeted

Received May 12, 2015; revision accepted May. 14, 2015.

For correspondence or reprints contact: Paul E. Kinahan, University of Washington Medical Center, 1959 N.E. Pacific St., Box 356004, Rm. NW040, Seattle, WA 98195-6004.

E-mail: kinahan@u.washington.edu

Published online Jun. 18, 2015.

COPYRIGHT (C) 2015 by the Society of Nuclear Medicine and Molecular Imaging, Inc.

DOI: $10.2967 /$ jnumed.115.159178 therapy (7). Similarly, an early ${ }^{18}$ F-FDG PET scan predicts response to tyrosine kinase inhibitors in gastrointestinal stromal tumors treated with imatinib (8) and lung cancers treated with gefitinib (9). Although these results and others support the use of ${ }^{18}$ F-FDG PET as both an integrated and an integral marker in trials of targeted therapy, well-designed prospective studies are needed to firmly establish this role for ${ }^{18}$ F-FDG PET (10). A key step in the validation of any biomarker is the evaluation of analytic validityin this case, the quantitative accuracy of the ${ }^{18} \mathrm{~F}-\mathrm{FDG}$ uptake measures. Specifically, what are the error bars in measurements from the PET images?

The study by Weber et al. (11) in this issue of The Journal of Nuclear Medicine presents needed data for PET to be usable in a clinical trial of new lung cancer studies. By pooling together prospective multicenter test-retest data from the American College of Radiology Imaging Network (ACRIN) 6678 and Merck MK0646-008 trials (74 patients total) in a rigorous analysis, the authors were able demonstrate the limits of normal variability of tracer uptake in multicenter imaging of advanced non-small cell lung cancer stages III-IV. These limits provide the framework for determining if there has been a response to therapy or if a measured change is due to variations to be expected in practice. There have been previous studies measuring the variability from test-retest studies (as noted by the authors), and although the variability observed in this study is slightly higher than in previous single-center studies, it is similar to the results of a previous carefully controlled multicenter test-retest study in 62 patients with gastrointestinal malignancies (12).

The test-retest results of such studies are needed to determine parameters for the use of quantitative PET/CT imaging for clinical trials and clinical care. Two other important components of such a framework are the definition of response criteria and the comprehensive standards for equipment, protocols, and quality assurance/quality control procedures. The former is provided by the continued development of the PET Response Criteria in Solid Tumors (13), which are supported by the results of this and previous studies. The standards are being provided by several international efforts, including the Quantitative Imaging Biomarker Alliance (14), which in cooperation with other groups (Society of Nuclear Medicine and Molecular Imaging, European Association of Nuclear Medicine, Radiological Society of North America, Food and Drug Administration, National Institute of Standards and Technology, manufacturers, pharma, and others) has developed the Uniform Protocol for Imaging in Clinical Trials (15) and the FDG PET/CT Profile. The Profile is a new type of document that affirms the measurement bias or precision under specific 
conditions and then specifies what is required to meet the level of measurement accuracy, which includes a subset of the Uniform Protocol for Imaging in Clinical Trials protocol as well as the entire instrumentation chain. Careful evaluation has revealed that the entire imaging chain needs quality assurance/quality control and that even the display stations used for analysis are subject to errors if not checked. Tools for quantitative imaging challenges developed through the National Cancer Institute's Quantitative Imaging Network (16) and Quantitative Imaging Biomarker Alliance are now being used for the entire acquisition and analysis chain.

Given the challenges with reproducibility and the pooling of data for meta-analysis in biomedical research (17), the sharing of data by Merck and ACRIN is a welcome model of progress in medical imaging research and a foundation for further progress in the development of the precision medicine paradigm. The study by Weber et al. (11) is both an exemplary illustration and a contribution to the necessary data needed to take full advantage of what quantitative molecular imaging can offer for cancer clinical trials, oncologists, and precision medicine for cancer patients (18).

\section{DISCLOSURE}

This study was supported by NIH grant U01CA148131. No other potential conflict of interest relevant to this article was reported.

\section{REFERENCES}

1. Siegel R, Ma J, Zou Z, Jemal A. Cancer statistics, 2014. CA Cancer J Clin. 2014;64:9-29.

2. Malakoff D. Clinical trials and tribulations: spiraling costs threaten gridlock. Science. 2008;322:210-213.

3. Haber DA, Gray NS, Baselga J. The evolving war on cancer. Cell. 2011; 145:19-24.
4. Doroshow JH, Kummar S. Translational research in oncology: 10 years of progress and future prospects. Nat Rev Clin Oncol. 2014;11:649-662.

5. Doot RK, McDonald ES, Mankoff DA. Role of PET quantitation in the monitoring of cancer response to treatment: review of approaches and human clinical trials. Clin Transl Imaging. 2014;2:295-303.

6. Weber WA, Petersen V, Schmidt B, et al. Positron emission tomography in nonsmall-cell lung cancer: prediction of response to chemotherapy by quantitative assessment of glucose use. J Clin Oncol. 2003;21:2651-2657.

7. Gebhart G, Gamez C, Holmes E, et al. ${ }^{18} \mathrm{~F}$-FDG PET/CT for early prediction of response to neoadjuvant lapatinib, trastuzumab, and their combination in HER2positive breast cancer: results from Neo-ALTTO. J Nucl Med. 2013;54:18621868.

8. Heinicke T, Wardelmann E, Sauerbruch T, Tschampa HJ, Glasmacher A, Palmedo H. Very early detection of response to imatinib mesylate therapy of gastrointestinal stromal tumours using 18fluoro-deoxyglucose-positron emission tomography. Anticancer Res. 2005;25:4591-4594.

9. Takahashi R, Hirata H, Tachibana I, et al. Early $\left[{ }^{18} \mathrm{~F}\right]$ fluorodeoxyglucose positron emission tomography at two days of gefitinib treatment predicts clinical outcome in patients with adenocarcinoma of the lung. Clin Cancer Res. 2012;18:220-228.

10. Mankoff DA, Pryma DA, Clark AS. Molecular imaging biomarkers for oncology clinical trials. J Nucl Med. 2014;55:525-528.

11. Weber WA, Gatsonis CA, Mozley PD, et al. Repeatability of ${ }^{18}$ F-FDG PET/CT in Advanced non-small cell lung cancer: prospective assessment in 2 multicenter trials. J Nucl Med. 2015;56:1137-1143.

12. Velasquez LM, Boellaard R, Kollia G, et al. Repeatability of ${ }^{18}$ F-FDG PET in a multicenter phase I study of patients with advanced gastrointestinal malignancies. J Nucl Med. 2009;50:1646-1654.

13. Wahl RL, Jacene H, Kasamon Y, Lodge MA. From RECIST to PERCIST: evolving considerations for PET response criteria in solid tumors. J Nucl Med. 2009;50(suppl 1):122S-150S

14. Buckler AJ, Bresolin L, Dunnick NR, Sullivan DC. A collaborative enterprise for multi-stakeholder participation in the advancement of quantitative imaging. Radiology. 2011;258:906-914.

15. Graham MM, Wahl RL, Hoffman JM, et al. Summary of the UPICT Protocol for FDG PET/CT imaging in oncology clinical trials. J Nucl Med. 2015;56:955-961.

16. Clarke LP, Nordstrom RJ, Zhang H, et al. The Quantitative Imaging Network: NCI's historical perspective and planned goals. Transl Oncol. 2014;7:1-4.

17. Ioannidis JP. How to make more published research true. PLoS Med. 2014;11: e1001747.

18. Collins FS, Varmus H. A new initiative on precision medicine. $N$ Engl J Med. 2015;372:793-795. 